\title{
Multichannel visible spectroscopy diagnostic for particle transport studies in the $\mathrm{H}-1$ heliac
}

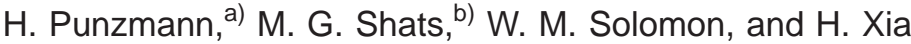 \\ The Australian National University, Canberra ACT 0200, Australia
}

(Presented on 10 July 2002)

\begin{abstract}
A multichannel spectroscopy diagnostic has been developed to study cross-field particle transport in the radiation-dominated low-temperature plasmas $\left(T_{e}<100 \mathrm{eV}\right)$ in the H-1 heliac. The optical setup covers the full plasma minor radius in the poloidal plane collecting light from ten parallel chords arranged tangentially to the flux surfaces. The light collected from the plasma is coupled into optical fibers and through interference filters into photomultipliers. Two such ten-fiber arrays are aligned parallel to one another to allow the simultaneous monitoring of two different spectral lines. The net radial electron particle flux is determined from the continuity equation by integrating over the ionization source term in the steady-state partially ionized plasma. The diagnostic measures the neutral line intensities and their ratios (in case of helium using the line ratio technique) and also measures excited neutral and ion spectral lines (in case of the argon plasma transport studies). A comparative analysis of the radial particle transport in the low- and high-confinement regimes is presented. (C) 2003 American Institute of Physics. [DOI: 10.1063/1.1537033]
\end{abstract}

\section{INTRODUCTION}

Understanding particle transport in magnetically confined plasmas is important in order to improve the confinement. Confinement bifurcations like the ones commonly observed in the H-1 heliac (e.g., Ref. 1) present convenient conditions for such studies. To study the electron particle flux in the radiation dominated, partially ionized, lowtemperature plasmas of $\mathrm{H}-1,{ }^{2}$ we use conventional line emission spectroscopy in a multichannel arrangement. The continuity equation

$$
\frac{\partial n}{\partial t}=-\nabla \cdot \Gamma+S
$$

relates the particle flux $\Gamma$ to the local particle source term $S$ defined mainly by the electron-impact ionization

$$
S=n_{e} n_{o}\left\langle\sigma V_{e}\right\rangle_{i}-n_{e} n_{i}\left\langle\sigma V_{e}\right\rangle_{r} .
$$

Here, $\left\langle\sigma V_{e}\right\rangle$ are the rate coefficients for the recombination (index $r$ ) and the ionization (index $i$ ) processes. In our case, the recombination term is small and can be neglected. The particle flux in a cylindrical coordinate system in steady state can be written as

$$
\Gamma(r)=\frac{1}{r} \int_{0}^{r} r n_{e}(r) n_{o}(r)\left\langle\sigma V_{e}\right\rangle_{i} d r .
$$

To estimate $\Gamma(r)$, radial profiles of the electron-density $n_{e}(r)$, neutral density $n_{o}(r)$, and electron temperature $T_{e}(r)$ are necessary. In the following section, we describe the diagnostic procedure that allows the required plasma param-

\footnotetext{
${ }^{a)}$ Electronic mail: horst.punzmann@anu.edu.au

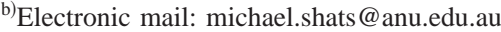

eters to be determined using the spectroscopy diagnostic, and show some results on the particle transport in different confinement regimes.

\section{DIAGNOSTIC SETUP AND METHOD}

Two linear fiber arrays whose images in the plasma are toroidaly displaced to collect light from ten radial separated chords as shown in Fig. 1. The matrix of $2 \times 10$ optical fibers is imaged into the plasma using camera zoom lenses $(f$ $=35-105 \mathrm{~mm}$ ), which allows changing the field of view without modifications to the hardware setup. Viewing chords are tangential with respect to the flux surfaces for the vertical view and cover the full plasma radius. The viewing chord diameter is about $16 \mathrm{~mm}(=0.17 a)$ and can be varied within the range of $\Delta r / a=0.05-0.4$ depending on the magnification. The two sets of ten optical fibers (quartz, $1 \mathrm{~mm}$ core diameter) are optically coupled to two interference filters to monitor two different spectral lines simultaneously. Alternatively, the filtering is achieved using two monochromators ( $f=0.3 \mathrm{~m}, 600 / 1200 / 2400$ grooves $/ \mathrm{mm}$ gratings) instead of the interference filters when the light throughput can be sacrificed for spectral line selectivity. The filtered light is then coupled to 20 photomultiplier tubes providing high-temporal resolution in the microsecond range.

To measure the electron particle flux $\Gamma(r)$, one needs to determine the required plasma parameters $n_{e}(r), T_{e}(r)$, and $n_{o}(r)$. Using the same spectroscopic hardware setup we use two different methods of calculating these quantities depending on the working gas, either helium or argon. The flowchart in Fig. 2 illustrates the analysis procedures for two different discharges.

In helium plasmas [Fig. 2(a)], a line intensity ratio method $^{3-7}$ is used to measure the electron temperature and density. Neutral helium spectral line ratios can be reliably interpreted using collisional-radiative models. ${ }^{8,9}$ This tech- 


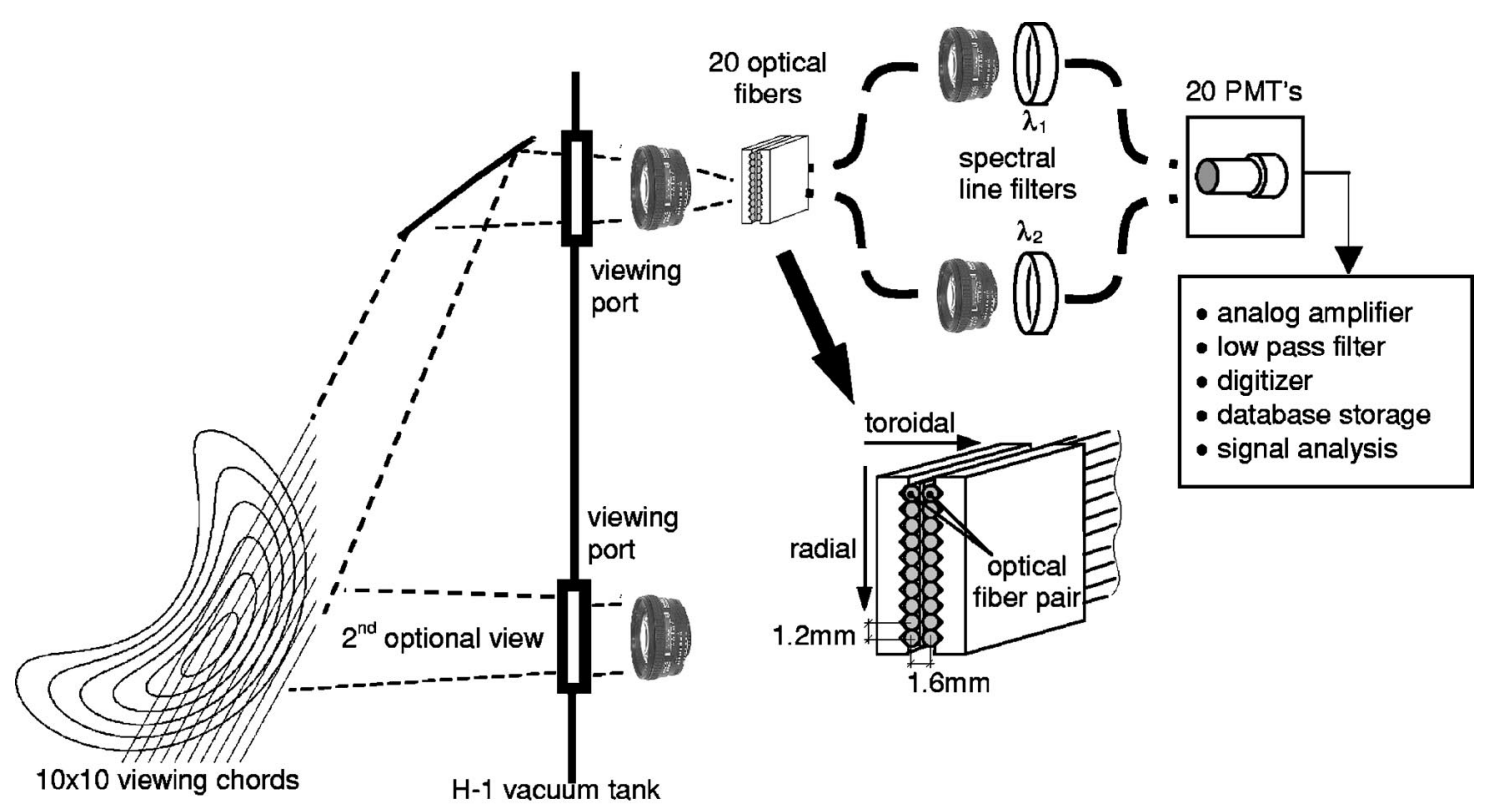

FIG. 1. Multichannel spectroscopy diagnostic setup with optional horizontal view and arrangement of $2 \times 10$ optical fiber matrix.

nique is used in helium plasmas with electron temperatures of up to $\sim 100 \mathrm{eV}$. We use intensity ratios of adjacent fiber pairs separated in the toroidal direction (see Fig. 1). Several suitable line ratios can be used, for example, the $T_{e}$-sensitive singlet/triplet pairs $\left(\lambda_{\text {singlet }} / \lambda_{\text {triplet }}[\mathrm{nm}]\right) 492.2 / 471.3,501.6 /$ $471.3,504.8 / 471.3$, and $728.1 / 706.5$ and the $n_{e}$-sensitive

\section{Helium plasma}

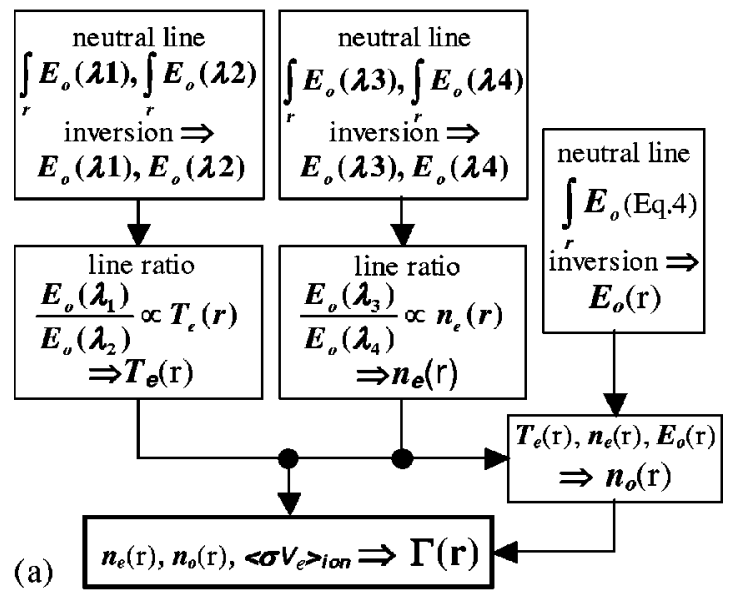

Argon plasma

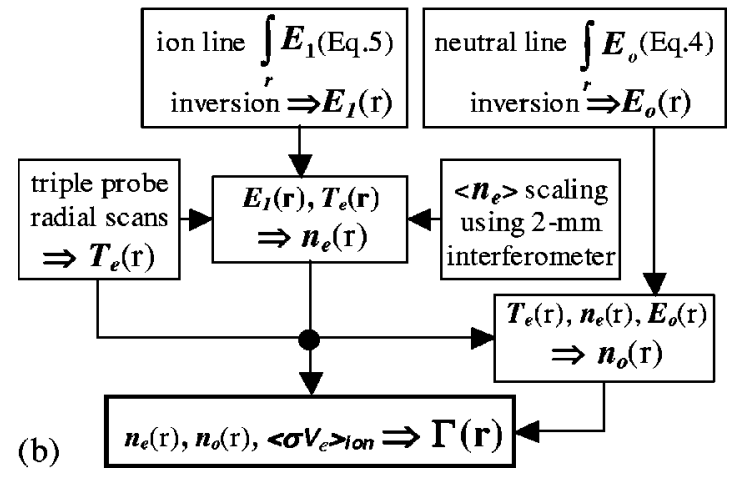

FIG. 2. Analysis procedure used in (a) helium and (b) argon plasmas. singlet/singlet pairs 492.2/504.8, 501.6/492.2, 501.4/504.8, and $667.8 / 728.1$. To recover the local density and temperature from the line-integrated measurements, conventional inversion techniques are used, which are based on the precomputed vacuum flux surfaces. The position of the last closed flux surface (LCFS) was also cross calibrated using radially scanable probes.

In argon plasmas [Fig. 2(b)], the chord averaged emissivity of the neutral and ion lines are used to calculate $n_{e}$ and $n_{o}$ using the following expressions. In the coronal approximation, which is applicable to our low-density discharges, the chord-averaged spectral line emission in the case of an excited neutral ${ }^{10}$ is given by

$$
E_{0}(p, q)=C_{0} \cdot \int_{0}^{l} \frac{n_{o}(r) \cdot n_{e}(r)}{T_{e}(r)^{1 / 2}} e^{\chi_{0}(p, q) / T_{e}(r)} d l
$$

where $C_{0}$ combines atomic constants (transition probability, line oscillator strength), $l$ is the viewing chord length, and $\chi_{0}(p, q)$ is the excitation potential of the transition $p-q$. A similar expression can be written for a singly charged ion line

$$
E_{1}=C_{1} \cdot \int_{0}^{l} \frac{n_{e}^{2}(r)}{T_{e}(r)^{1 / 2}} e^{\chi_{1} / T_{e}(r)} d l
$$

where $C_{1}$ is an atomic constant and $\chi_{1}$ the ionization potential. Using Eq. (5) in combination with the local temperature profiles from the triple probes, we calculate $n_{e}(r)$ after the radial inversion of the chord averaged emissivity $E_{1}$. The $n_{e}(r)$ profiles are then scaled using the line average density measured by a $2 \mathrm{~mm}$ interferometer. Similarly, we can now compute the neutral density $n_{o}$ using Eq. (4). First the neutral 


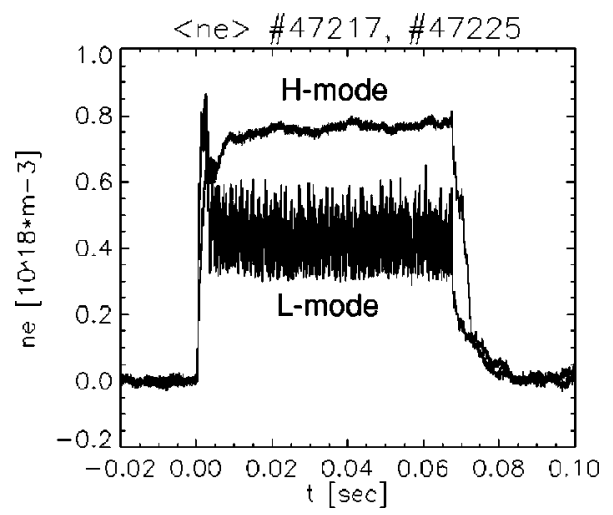

FIG. 3. Chord-averaged electron density in the $\mathrm{L}$ and quiescent $\mathrm{H}$ mode.

line emissivity $E_{o}$ is radially inverted and then $n_{o}(r)$ is computed using $n_{e}(r)$ and $T_{e}(r)$.

\section{EXPERIMENTAL RESULTS}

Experiments have been performed on the $\mathrm{H}-1$ helical axis stellarator having a major radius of $R_{0}=1.0 \mathrm{~m}$ and a minor radius of less than $0.2 \mathrm{~m}$. The experiments in argon plasmas were performed at low-magnetic fields $(B<0.2 \mathrm{~T})$ using $7 \mathrm{MHz}$ radio frequency heating at power levels of less than $100 \mathrm{~kW}$. Typical argon plasma parameters during the experiments were average electron density $n_{e}=(0.5-1.0)$ $\times 10^{18} \mathrm{~m}^{-3}$, electron temperature $T_{e}=(5-40) \mathrm{eV}$, and ion temperature $T_{i}=(20-60) \mathrm{eV}$.

The argon plasma at low fields $(B<0.07 \mathrm{~T})$, which we refer to as $L$ mode, is dominated by large coherent fluctuations. This can be seen on the lower-time trace $\left(\left\langle n_{e}\right\rangle \approx 0.45\right.$ $\left.\times 10^{18} \mathrm{~m}^{-3}\right)$ of the electron density signal in Fig. 3. An increase in the magnetic field leads to a transition to a confinement regime with significantly increased density and suppressed fluctuations. The upper-time trace in Fig. 3 shows the electron density during the so-called quiescent $\mathrm{H}$ mode $\left(\left\langle n_{e}\right\rangle \approx 0.75 \times 10^{18} \mathrm{~m}^{-3}\right)$.

Reconstructed electron-density profiles from the spectroscopic diagnostic in these two confinement modes are shown in Fig. 4(a). The centrally peaked L mode profiles of the electron-density changes across the L-H transition to slightly hollow profiles in $\mathrm{H}$ mode, with a significant increase in the density gradient in the outer half of the plasma radius. The comparison of these profiles with measurements of the probe ion saturation current $I_{\text {sat }}$, shows good agreement assuming a flat ion temperature profile. Note that $I_{\text {sat }} \propto n_{e}\left(T_{e}+T_{i}\right)^{1 / 2}$ and $T_{i}>T_{e}$ in H-1. ${ }^{2}$ Figure 4(b) shows the neutral density profile for $\mathrm{L}$ and $\mathrm{H}$ modes. The neutral density decreases by a factor of 2-5 from the edge to the center in the argon discharges. The estimated $n_{e}$ error of $15 \%$ in Fig 4(a) is an accumulation of errors in the relative channel intensity calibration, the error in the computation of the local emissivity during the inversion process, and the tolerance in the measurements of the probe $T_{e}$ and the interferometer $n_{e}$. The same applies for the neutral density error, which can be up to $20 \%$ in Fig 4(b).

The radial profile of the particle flux $\Gamma(r)$ [Eq. (3)] is determined using in a wide range of magnetic fields, $B$ $=(0.04-0.13) \mathrm{T}$, covering both $\mathrm{L}$ and $\mathrm{H}$ modes. We use the
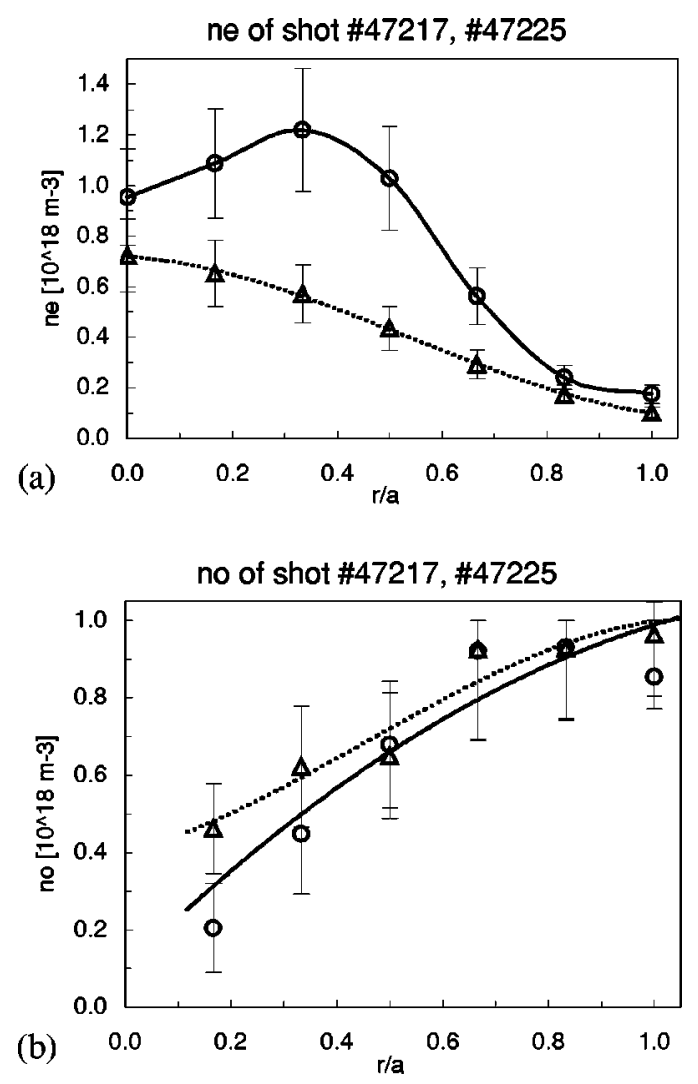

FIG. 4. Reconstructed (a) electron-density profiles $n_{e}(r)$ and (b) neutral density profiles $n_{o}(r)$ for $\mathrm{L}$ mode (doted line) and $\mathrm{H}$ mode (solid line).

effective diffusion coefficient, $D_{\text {eff }}=\Gamma(r) /(d n / d r)$, to characterize the transport properties of these discharges. Shown in Fig. 5 are the radial profiles of $D_{\text {eff }}$ computed for many $\mathrm{L}$ mode (triangle) and $\mathrm{H}$ mode discharges during a magneticfield scan. The particle transport in the inner half of the plasma radius appears to be similar for $\mathrm{L}$ and $\mathrm{H}$ modes, up to about 0.45 of the plasma radius. The large scatter of the data points in this region points is due to the division of the flux $\Gamma$ by small $(\sim 0)$ density gradients, since the $\mathrm{H}$ mode

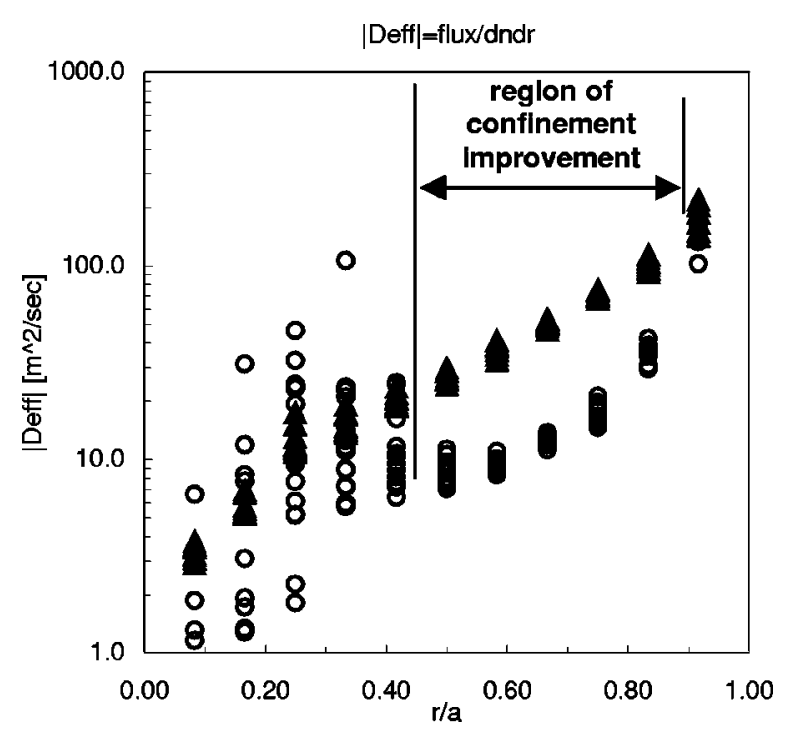

FIG. 5. Effective diffusion coefficient $\left|D_{\text {eff }}\right|$ as a function of normalized radius $r / a$ in $\mathrm{L}$ mode (triangles) and $\mathrm{H}$ mode (circles) discharges. 
electron-density profiles are flat in the center. In the outer half of the plasma, a large reduction in the diffusion coefficient is observed. The confinement improvement during the transition from $\mathrm{L}$ to $\mathrm{H}$ mode is mainly localized to the outer half of the plasma radius. This correlates with the fact that the fluctuation-driven particle flux in these L mode discharges is strong only in the outer half of the plasma radius.

Summarizing, a flexible multichannel spectroscopic diagnostic has been developed for the $\mathrm{H}-1$ heliac to study partially ionized plasmas in different working gases. The electron and neutral density profiles in argon plasmas have been measured, and have been used to compute radial particle fluxes. The diagnostic has been successfully used to characterize the transport coefficients in low- and high-confinement regimes in $\mathrm{H}-1$.
${ }^{1}$ S. M. Hamberger, B. D. Blackwell, L. E. Sharp, and D. B. Shenton, Fusion Technol. 17, 123 (1990)

${ }^{2}$ M. G. Shats, C. A. Michael, D. L. Rudakov, and B. D. Blackwell, Phys. Plasmas 5, 2390 (1998)

${ }^{3}$ S. P. Cunningham, USA Energy Comm. Report No. Wash.-289, 1955, p. 279.

${ }^{4}$ R. H. Huddlestone and S. L. Leonard, Plasma Diagnostic Techniques (Academic New York, 1965), p. 254.

${ }^{5}$ S. Sasaki, Rev. Sci. Instrum. 67, 3521 (1996).

${ }^{6}$ A. R. Field and P. G. Carolan, Rev. Sci. Instrum. 70, 355 (1999).

${ }^{7}$ N. Brenning, J. Quant. Spectrosc. Radiat. Transf. 24, 293 (1980).

${ }^{8}$ T. Fujimoto, J. Quant. Spectrosc. Radiat. Transf. 21, 439 (1979).

${ }^{9}$ R. H. Huddlestone and S. L. Leonard, Plasma Diagnostic Techniques (Academic, New York, 1965), p. 208.

${ }^{10}$ M. G. Shats, D. L. Rudakov, B. D. Blackwell, G. G. Borg, R. L. Dewar, S. M. Hamberger, J. Howard, and L. E. Sharp, Phys. Rev. Lett. 77, 4190 (1996). 